\title{
Le Portique
}

Revue de philosophie et de sciences humaines

43-44 | 2019

Japon voyages intérieurs

\section{L'expérience mystique du rire}

\section{Sylvie Paillat}

\section{OpenEdition}

Journals

Édition électronique

URL : http://journals.openedition.org/leportique/3627

DOI : $10.4000 /$ leportique.3627

ISSN : $1777-5280$

\section{Éditeur}

Association "Les Amis du Portique"

\section{Édition imprimée}

Date de publication : 1 septembre 2019

Pagination : 291-316

ISBN : 978-2-916332-40-6

ISSN : 1283-8594

\section{Référence électronique}

Sylvie Paillat, "L'expérience mystique du rire », Le Portique [En ligne], 43-44 | 2019, document 21, mis en ligne le 10 février 2020, consulté le 25 mars 2021. URL : http://journals.openedition.org/leportique/ 3627 ; DOI : https://doi.org/10.4000/leportique.3627

Ce document a été généré automatiquement le 25 mars 2021.

Tous droits réservés 


\title{
L'expérience mystique du rire
}

\author{
Sylvie Paillat
}

Je ne suis à la vérité que le rire qui me prend. ${ }^{1}$

Dans le roman Le Nom de la rose d'Umberto Eco, dont l'intrigue se déroule en l'an 1327, dans une abbaye bénédictine du nord de l'Italie, des moines sont retrouvés morts dans des circonstances suspectes. Un des franciscains les plus importants, Guillaume de Baskerville, disciple des théologiens anglais Roger Bacon et Guillaume d'Ockham, accompagné du jeune Adso de Melk, est chargé de mener l'enquête sur ces morts mystérieuses dont l'énigme tourne autour de la disparition du tome II de La Poétique d'Aristote, traitant de la comédie et du rire. Le moine bénédictin Jorge de Burgos se méfie du rire qu'il considère comme diabolique et dont il a peur parce qu'il y voit les forces destructrices du mal et de la négation. Guillaume de Baskerville quant à lui, appartient à l'ordre des frères mineurs fondé par saint François d'Assise qui préconise à ses disciples la gaité et le visage rieur alors que règne l'austérité dans la plupart des monastères ; ce dont témoigne Jorge au contraire du franciscain, précurseur d'Erasme et de Rabelais qui y voit un instrument d'émancipation intellectuelle et spirituelle, « le rire libère le vilain de la peur du diable (...) Se libérer de la peur du diable est sapience (...) Le diable est l'arrogance de l'esprit, la foi sans sourire, la vérité qui n'est jamais effleurée par le doute. $»^{2}$ Le rire est donc selon le vieux moine agélaste Jorge, l'œuvre du diable, la manifestation du péché d'orgueil et celle de la faiblesse de la chair qu'il faut par conséquent interdire tout comme la Poétique II d'Aristote. On ignore tout de l'existence ou non de cet ouvrage mais Umberto Ecco suppose dans son roman que cette œuvre a été réellement écrite. Elle aurait été l'objet d'un autodafé ou d'une disparition suite aux stricts interdits monacaux, notamment ceux édictés par Benoît concernant le rire. L'absence de l'œuvre d'Aristote au sein même de cette abbaye, évoque cette absence/présence du rire dont on peut dire que la nature mystique, c'està-dire mystérieuse et secrète (selon les étymologies latine et grecque), évoque l'absence/présence de Dieu et soulève un problème d'ordre théologique qui préoccupe en partie le Christianisme médiéval. En effet, le rire devient un véritable sujet de discussion et de questionnement relevant de la casuistique, notamment au treizième siècle où un débat public, un quod libet est organisé à l'Université de Paris autour des problèmes qu'il soulève. On revient par exemple sur cette révélation que Jésus n'aurait 
jamais ri et on pose cette contradiction que s'il s'est incarné en devenant homme, il a par conséquent entièrement revêtu la nature humaine, ce qui revient à dire qu'il aurait pu rire puisqu'on admet que le rire est une spécificité exclusivement humaine.

Or, s'il n'a pas ri, peut-on pour autant en déduire que le rire est bien l'œuvre du diable, celui qui divise et dont la double nature conduit à la tromperie, l'imposture, la négativité et le mal ? En ce sens, le rire serait anti-mystique. On peut d'une part entendre par ce terme, ce qui s'oppose à la mystique chrétienne dogmatique et institutionnelle, fondée sur une théologie rationnelle spéculative. Anti-mystique signifie donc ici anti-institutionnel et anti-théologique. D'autre part, dans un sens plus restreint, si la mystique est l'expérience intime de l'absolu, l'union intime avec Dieu qui implique une foi profonde et suppose une ascèse, la nature physiologique, souvent disgracieuse, excessive et transgressive $d u$ rire tout autant que sa fonction démystificatrice, pourfendeuse de toute croyance fondée sur la crainte et l'ignorance, ne pourrait qu'être anti-mystique, c'est-à-dire profane et athée. À moins qu'il ne faille, au contraire, considérer le rire comme un signe singulier de l'expérience mystique, un don de Dieu que le corps reçoit mystérieusement en lui et qui témoigne en son paroxysme de l'amour et de la joie. Excédant largement la raison humaine mais aussi le corps, sa nature irrationnelle et libre, tout autant que celle du mystique, pourrait conduire à l'extase. On pourrait dès lors parler d'un rire mystique, d'une expérience mystique du rire qui ne serait plus tout à fait le propre de l'homme, en tant que grâce divine. Il serait l'impropre de l'homme. Il ne lui appartiendrait plus.

3 On peut ainsi dire que Jorge représente la négation d'un tel rire, refusant sa part spirituelle et critiquant sa fonction démystificatrice, tandis que Guillaume de Baskerville, certes davantage théologien positif du rire que mystique - il nous appartiendra en première partie de revenir sur cette distinction conceptuelle entre la théologie et la mystique, la théologie étant un terme plus générique, disons un englobant de la mystique - la défend et permet néanmoins de comprendre cette étrange et protéiforme manifestation d'un rire qu'on pourrait qualifier de mystique. L'Ambiguïté du rire à la fois critique et destructeur, spirituel et corporel, intérieur et extérieur, divin et diabolique, ouvert et fermé, joyeux et cynique, aussi banal soit-il, n'est-il donc pas le révélateur de l'ambiguïté même de l'expérience mystique, tout autant extraordinaire qu'ordinaire, surnaturelle que naturelle ? Certaines similitudes entre eux, dont cette expérience singulière du corps comme passage à la limite, à la fois étranger et familier, intime et " extime " selon l'expression lacanienne, laisserait entendre que cette mystique ou union directe de l'esprit humain avec Dieu, peut se passer dans le rire. Pourtant, c'est paradoxalement par le refus du corps comme lieu de jouissance, du moins pour certaines de ses manifestations excessives, qu'une orthodoxie chrétienne fondée sur une théologie rationnelle et institutionnelle manichéenne, notamment héritée de la théorie des Pères de l'Église grecque, l'a rejeté, jetant ainsi le bébé avec l'eau du bain, tout autant qu'elle a été conduite à rejeter une certaine mystique, comme connaissance expérimentale et singulière de l'union directe à Dieu. La mystique et le rire s'inscriraient donc dans l'hétérodoxie, voire l'hérésie. On peut même aller jusqu'à se demander si parmi les sorcières condamnées au bûcher pour hérésie, il n'y avait pas des mystiques "sauvages ${ }^{3}$ selon le terme et le titre d'un ouvrage empruntés à Michel Hulin. Leur étrange comportement, proche de l'hystérie et de la folie, dont les rires et soubresauts ébranlant leur corps témoignent, pourrait en effet, laisser penser à une transe extatique. 
4 On procédera donc tout d'abord à l'examen de ce qui justifie, notamment à l'époque médiévale, ce rire anti-mystique ou rire de mauvaise foi. Pourtant, loin d'être un antagoniste de la mystique, le rire pourra être envisagé comme rire de la joie, de la bonne foi et comme disposition mystique singulière dont témoignent certaines figures, celle d'Isaac, fils d'Abraham et de Sara dans l'ancien testament, figure symbolique fondatrice d'une joyeuse espérance, et celle du mystique François d'Assise. Ainsi, le rire mystique ne serait pas la marque de la supériorité et de l'orgueil humain. C'est au contraire par lui que s'effaceraient progressivement le moi et ses résistances égotiques, devenu humble jusqu' au rien, pour se fondre en Dieu ou Dieu en lui. Mourir à soi par le rire serait par conséquent aux antipodes de la mortification que s'imposent tout autant religieux que mystiques, si l'on s'en tient à la théologie canonique fondée sur la pratique de l'ascèse. Quelle est donc cette ascèse singulière à la mystique qui ne renie pas le corps mais qui s'en sert comme d'un lieu de retrait et d'ouverture à Dieu ? Le corps mystique est un corps non clos dont la porosité et l'ouverture peuvent passer par le rire. Comme " pas-de-sens » pour reprendre la formule lacanienne, le rire serait donc rencontre avec le réel pur où règne le rien. Il serait un passage entre le corps physique et le corps spirituel. C'est paradoxalement par la référence à Bataille dans L'expérience intérieure que le rire est le mieux analysé dans sa dimension mystique. Son " athéologie rieuse » semble finalement épouser - au-delà de l'aspect transgressif du rire que l'on retient habituellement dans la pensée irréligieuse de Bataille, entre mysticisme et démystification - cette expérience singulière du corps et du rire chez le mystique.

\section{Le rire anti-mystique ou le rire de mauvaise foi}

5 Le Nom de la rose qui nous sert d'assise et de fil conducteur dans ce travail afin de comprendre le rejet du rire comme antimysticisme et manifestation de la mauvaise foi, s'inscrit dans ce contexte médiéval et chrétien où l'on voit progressivement se distinguer et s'opposer une théologie spéculative fondant l'orthodoxie chrétienne incarnée par l'Église et une théologie mystique comme forme particulière de l'expérience religieuse. Toutefois, Guillaume de Baskerville n'est ni tout à fait la figure de l'orthodoxie chrétienne davantage représentée dans le roman par le dominicain et inquisiteur Bernardo Gui et par l'obscurantiste Jorge, ni tout à fait la figure du mystique. Théologien positif, c'est-à-dire cataphatique, scolastique nominaliste, comme son maître Guillaume d'Ockham, son approche de Dieu reste savante et rationnelle. Elle annonce du reste l'esprit humaniste d'un Rabelais et d'un Érasme dont le doute va égratigner les dogmes chrétiens institutionnalisés, notamment ceux des Pères de l'Église dont les bénédictins suivent l'enseignement.

6 Il faut préciser que le terme mystique existe déjà et qu'il est antérieur à la chrétienté. Pour l'antiquité grecque notamment, il désigne ce qui est caché et mystérieux dans la nature. Le mystère est immanent tandis que pour le christianisme naissant, le mystère est de nature transcendantale. Il devient mystère de la trinité et mystère de Christ ressuscité, notamment chez les Pères de l'Église dont saint Paul est le représentant. On doit précisément l'invention de " la mystique " comme doctrine et expérience religieuse singulière, au pseudo-Denys l'Aréopagite. Il ne parle pas à proprement parler de la mystique mais de la théologie mystique qu'il distingue de la théologie symbolique et de la théologie spéculative. Si l'une et l'autre impliquent une démarche rationnelle et une approche cataphatique en s'appuyant notamment sur la symbolique et le récit biblique, il considère la théologie mystique comme le degré suprême de la connaissance 
de Dieu, une connaissance supra-intellectuelle dont l'approche est apophatique. En d'autres termes, la mystique est inhérente à une théologie négative niant que l'on puisse définir Dieu par des termes positifs et les remplaçant par des termes négatifs. De Dieu, on ne peut rien dire : «Plus haut nous portons notre regard, plus s'abrège aussi notre discours lorsqu'il embrasse d'un seul coup d'œil les intelligibles. De même, maintenant que nous allons pénétrer dans la Ténèbre qui est au-delà de l'intelligible, nous ne trouverons pas seulement des paroles plus concises, mais jusqu'à leur absence et perte du sens. $»^{4}$ Afin de préparer l'âme à cette union avec Dieu, catharsis et ascèse sont nécessaires. Elles lui permettent d'accéder au dépouillement, de devenir pure et simple, de la libérer du corps matériel pour en découvrir sa dimension spirituelle ou bien pour découvrir un autre corps (dans le bouddhisme il y a trois dimensions corporelles ou trois corps. Le premier est matière, le deuxième est éther, le troisième est aura). Pseudo-Denys exhorte Timothée à suivre cette voie entre le monde sensible et le monde intelligible :

"Quant à toi, mon cher Timothée, exerce-toi sans relâche aux contemplations mystiques, abandonne toutes sensations et jusqu'aux spéculations de l'intelligence, laisse tout le sensible, tout l'intelligible, tout l'être et le non-être; ainsi, autant que tu en es capable, tu seras surélevé par la voie de l'inconnaissance jusqu'à ne plus faire qu'un avec celui qui est au-delà de toute essence et de toute connaissance. $»^{5}$

Dans ces conditions, on comprend que le rire comme manifestation corporelle parfois excessive et bruyante, ne puisse préparer à une disposition spirituelle ou une expérience ascétique. Il témoigne au contraire de la chute de l'humanité suite au péché originel et il est la manifestation du diable pour le christianisme. De plus, la représentation du Christ qui n'aurait jamais ri, confirme la thèse d'un rire antimystique. Ce dernier est d'autant plus le propre de l'homme qu'il s'exprime dans sa nature la plus bassement matérielle. Le rire est obscène et bestial, tout comme la sexualité peut l'être. D'où son rejet et sa condamnation par le vieux moine Jorge : «Le rire est un souffle diabolique, qui déforme les linéaments du visage et fait ressembler l'homme au singe $\aleph^{6}$. Pourtant, ceux-ci ne datent pas de l'époque médiévale mais sont un héritage de l'antiquité grecque et notamment de la philosophie platonicienne. Ils s'inscrivent pour partie dans ce modèle ascétique qui se poursuit dans le Christianisme avec un certain courant agélaste fondé sur l'autorité des Pères de l'Église grecque dont le bénédictin Jorge est le garant. Ce dernier suit la règle du Maître, un modèle dont on pense qu'il a peut-être été édicté par saint Benoît lui-même. Il permet de comprendre la vie quotidienne dans les monastères de l'époque. L'historien Jacques Le Goff, dans son étude sur Le rire dans les règles monastiques du haut Moyen Âge ${ }^{7}$, montre d'une part ce détournement du sens charnel des textes fondamentaux du Christianisme; d'autre part, il justifie qu'au lieu même de cette règle du maître :

"On a surtout retenu les textes hostiles au corps, de type ascétique, telle la fameuse phrase de Grégoire le Grand définissant le corps comme l'abominable vêtement de l'âme. La Règle du Maître explique en fait que le corps peut être le lieu de la malédiction comme le lieu du salut de l'âme. Dans les deux sens, que ce soit de l'extérieur vers l'intérieur ou de l'intérieur vers l'extérieur, le corps humain dispose de filtres : les trous du visage; les yeux, les oreilles et la bouche sont les filtres du bien et du mal et doivent être utilisés de façon à laisser entrer ou s'exprimer le bien et à barrer la route au mal. La Règle du Maître parle du «verrou de la bouche ", de "la barrière des dents ", etc. Quand le rire s'apprête à fuser, il faut empêcher absolument que ce rire ne s'exprime; et l'on voit comment, de toutes les formes mauvaises d'expression qui viennent de l'intérieur, le rire est la pire : la pire souillure de la bouche. Tout ceci est lié à une sorte de physiologie chrétienne, derrière laquelle d'ailleurs on reconnaît des traités médicaux, des croyances physiologiques si l'on peut dire. " $^{8}$ 

immoralité, à la manifestation du diable. En témoignent les représentations artistiques picturales et sculpturales dont les tympans des églises romanes et gothiques sont pourvus, ainsi que les nombreuses gargouilles des cathédrales montrant le faciès d'un diable ricaneur. Le rire s'inscrit donc dans le corps et par ses manifestations hybristiques, il le déforme. D'où ce rapprochement avec le diable.

9 L'invention du diable comme figure menaçante est aussi pour l'Église le levier du pouvoir qui permet de contrôler les fidèles et d'empêcher toute rébellion possible, notamment par le côté subversif du rire. Le rire est donc lui aussi sous le contrôle de l'Église. C'est pourquoi celui-ci fait l'objet d'interdiction stricte, surtout dans les monastères où une législation rigoureuse est établie, qui dès lors crée la culpabilité et le nécessaire devoir de punition ou d'autopunition pour quiconque transgresserait cet interdit. L'historien Georges Minois précise que «La plupart des règles prévoient des châtiments contre les moines qui seraient surpris à rire ou à plaisanter. Dans celle dite des quatre pères, composée à Lérins vers 400-410, on lit : Si quelqu'un est surpris à rire ou à dire des plaisanteries (...) nous ordonnons que, durant deux semaines, un tel homme soit, au nom du Seigneur, réprimé de toute façon par le fouet de l'humilité »" Et en effet, si le rire est particulièrement prohibé dans les monastères, c'est parce qu'il y est assimilé à un manque d'humilité, première vertu cardinale ainsi que l'observation du silence pour être moine et éviter tout péché par orgueil. C'est l'argument dont use Jorge contre Guillaume de Baskerville. De plus, dans le milieu monastique, les moines sont en majorité persuadés que "l'enfer est le lieu du rire. Au VIII siècle, Bède le Vénérable rapporte le récit d'un certain Drycthelm, ressuscité, qui a fait un séjour chez Satan où il a entendu un rire terrible, comme si une populace se moquait d'ennemis

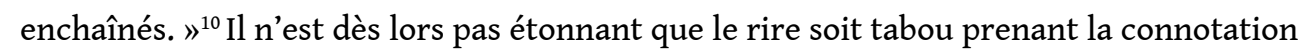
négative de ce qui est impur, impie et effrayant. Ce tabou du rire lié au diable ne repose pas sur un motif rationnel ou moral. Les sanctions qui découlent de toute transgression sont normalement acceptées parce qu'émanant d'abord de l'ordre du surnaturel, ce qui renforce le pouvoir des hommes d'Église dont les fidèles croient qu'ils l'ont reçu de Dieu. Par conséquent, les hommes d'église sont pour ceux-ci, les protecteurs des forces surnaturelles maléfiques. Dans Rire au Moyen Âge ${ }^{11}$, on apprend que le plus célèbre et influent des législateurs grec, saint Basile, avec l'aide de Jean Chrysostome, le plus agélaste des pères de l'Église, introduit une suite de petites et grandes règles par le grand argument contre le rire : « Jésus, modèle que le chrétien doit imiter, n'a pas, durant sa vie terrestre, ri une seule fois, comme l'atteste l'évangile » Et effectivement retrouve-t-on dans une des grandes règles (chapitre 16-17) de saint Basile citant l'évangile selon saint Luc que Jésus n'aurait jamais cédé au rire. Au contraire, il aurait proclamé malheureux ceux qui se laissent dominer par le rire. La réforme des ordres monastiques qui conduit à un ascétisme rigoureux dans lequel l'angélisme domine, ne concerne pas uniquement le rire mais s'étend à tout le corps, si bien que, remarque Jacques Le Goff : « L'intérêt en effet des attitudes théoriques et pratiques à l'égard du rire me paraît résider pour l'historien en grande partie dans le caractère mixte du rire, phénomène culturel qui s'exprime à travers le corps et dont l'étude éclaire aussi l'histoire des attitudes à l'égard du corps. ${ }^{12}$ Le Christianisme est donc traversé par cette contradiction où il affirme tout à la fois par l'incarnation, l'importance du corps comme lieu possible de rédemption et des attitudes théoriques et pratiques anticorporelles. Ainsi, y a-t-il un courant théologique qui s'inscrit contre le corps et le rire 
au Moyen Âge. La superstition n'en est pas exempte. Le vieux moine Jorge en témoigne. Ce courant négateur va à l'encontre d'une théologie positive et rationnelle, celle-là même qu'incarne Guillaume de Baskerville qui défend la raison critique et la connaissance contre l'obscurantisme. Il tente donc de convaincre les moines Bénédictins en leur prouvant que les Saintes Écritures n'ont jamais interdit le rire et que le rire est une spécificité humaine. Tout au long de ce roman, l'ordre franciscain que représente Guillaume de Baskerville est décrit comme plutôt ouvert d'esprit et riche de sciences. Ce dernier s'oppose à l'austérité de l'ordre bénédictin. Le rire y est souvent l'objet de discussions violentes entre les moines, et ce sujet définit à lui seul la vrai thématique de ce roman : La diabolisation du rire contre la liberté d'agir et la notion de liberté dans les ordres. Par cette diabolisation, le rire apparait donc comme l'adversaire suprême, objet de haine d'autant plus important qu'il s'enracine dans une haine du corps conduisant au déni du rire par la contemption du corps. Le rire est donc peu orthodoxe et ne peut être qu'anti-mystique au regard d'une théologie spéculative et mystique chrétienne canoniques.

10 Saint Bernard et la mystique Hildegarde von Bingen, deux figures religieuses et spirituelles incontestables pour le modèle chrétien, sont des exemples notoires en tant que contempteurs et agélastes du rire. Leurs invectives et diatribes contre le rire se justifien-t néanmoins en partie par ce mauvais rire, rire transgressif, rire de la mauvaise foi et de la méchanceté. En effet:

"Hildegarde raconte qu'elle a été objet de moqueries quand elle est tombée malade. Depuis, quand elle veut imposer une réforme austère, elle attribue à Satan cette dérision. Dans sa première biographie, la Vita sanctae Hildegardis des moines Godefricus et Theodoricus, elle est aux prises avec un démon moqueur, qui possède une femme et qui l'affuble du sobriquet scrumpilgardis, la vieille ratatinée. Le même démon moqueur, appelle Bernard, Bernardinet à cause de sa fragilité physique. $»^{13}$

11 On retrouve dans la moquerie une des raisons fondamentales justifiant la condamnation du rire depuis l'antiquité, appelé mauvais rire en raison de son caractère pervers et destructeur. Mais, pour Hildegarde et Bernard, la condamnation du rire porte sur une haine généralisée du rire particulièrement pour la raison qu'il "s'enracine dans les parties dégoûtantes et honteuses du corps " ${ }^{14}$. Chez Hildegarde, le rire est en effet «le jet d'un phallus qui éjacule par saccades »" Elle précise que «le corps est secoué par le rire comme par les mouvements de la copulation, et, au moment de la plus grande jouissance, le rire fait jaillir les larmes comme le phallus fait jaillir le sperme $»^{16}$. Saint Bernard, quant à lui, se contente de comparer le rire à " une vessie trop gonflée, secouée en tous sens par l'air qui s'échappe $»^{17}$. Pour ce dernier, le rire en vient même à être le résultat d'efforts inutiles puisque la véritable vertu est dans le refus du rire « qui distingue d'ailleurs chrétiens et païens, car eux seuls savent qu'il faut pleurer dans l'attente du jugement, ce qui leur donne évidemment un avantage considérable. ${ }^{18}$ Leur mépris du corps, du rire ici associé à la sexualité, se justifie néanmoins si l'on s'en tient à l'orthodoxie chrétienne et à l'ascèse qu'elle implique.

12 Il faut enfin dire que l'irrationalité du rire qui jaillit de manière imprévisible et chaotique, a souvent été associée à l'hystérie et à la folie. La mystique, dans son expérience limite du corps et de l'esprit peut également y faire penser. Ainsi, le rire est d'autant plus anti-mystique qu'il est le "fou-rire ", rire aux éclats de la raison, rire pathologique, asocial et marginal eu égard à toute forme d'institution. Dans La fable mystique Michel de Certeau analyse sous un double regard, celui du christianisme et celui de la psychanalyse lacanienne, les figures de la mystique au seizième et dix- 
septième siècles. Il axe notamment ses recherches sur ceux qui sont en marge de l'institution. Parmi eux, les pauvres, les idiots, les fous mystiques, « ceux qui rient ${ }^{19}$. On pourrait rajouter à cette liste, les sorcières dont le rire diabolique hante l'imaginaire des enfants et adultes. Qui étaient-elles donc, pour la plupart condamnées au bûcher à l'époque médiévale et surtout au seizième et dix- septième siècles? De véritables sorcières, des mystiques sauvages, des hystériques, de pauvres bougresses ou des femmes atypiques dont l'originalité les faisait être clowns, c'est-à-dire de singulières et drôles de figures féminines en marge de la société et des institutions ? Quelles que soient leurs figures, les mystiques deviennent suspects, parce qu'ils se démarquent de la mystique chrétienne encore enracinée dans un discours théologique. L'on voit ainsi émerger, en dehors de toute théologie canonique et institutionnelle chrétienne dominant en majeure partie le Moyen Âge, une mystique plus naturelle et " sauvage» ${ }^{20}$ qui devient l'affaire d'une foi personnelle, c'est-à-dire la connaissance et l'expérience singulières et subjectives d'une réalité absolue, transcendante ou divine. Michel de Certeau constate justement ce glissement sémantique du terme et l'invention, à proprement parler de " la » mystique : "Au croisement du XVI et XVII siècles, le théologien mystique devient "un mystique" ou "une mystique", puis à son tour, "la théologie mystique" devient "la mystique". [...] Le substantif mystique semble faire son apparition dans les milieux ou à propos des groupes qui s'éloignent le plus de l'institution théologique et, comme beaucoup de noms propres, il a d'abord forme de sobriquet ou d'accusation. ${ }^{21}$ Apparaît donc aux yeux de la société et des institutions, à cette époque versée dans le classicisme, une mystique irrationnelle peu crédible et peu sérieuse, une sorte de mystification dont il faut se méfier tout comme le rire. Apparenté à la folie, le rire est par conséquent rejeté, de la même manière qu'il l'était au Moyen Âge. Il ne peut être qu'anti-mystique, quand bien même à la Renaissance, il se trouve revalorisé, notamment grâce à l'humaniste François Rabelais qui en fait une spécificité humaine de premier ordre lorsque commence à s'effondrer toute valeur transcendante.

Pourtant, même si encore sous l'influence de la théologie mystique chrétienne aux seizième et dix-septième siècles, le rire mystique ne peut pas être considéré, même si le rire et le corps sont diabolisés, notamment pour ce qui est du Haut Moyen Âge et du Moyen Âge central ou classique, il n'en reste pas moins que toute l'époque médiévale est paradoxalement le véritable âge d'or du rire, ce qui montre un certain écart entre le discours et la réalité. Cet écart se retrouve d'une part dans cette distinction entre l'ordre régulier, l'ordre séculier et le peuple pour lequel le rire a sa place. C'est pourquoi, constatant que le rire ne peut être supprimé parce qu'il fait partie de la tradition populaire, l'Église le ritualise par l'institution des fêtes, certaines religieuses, d'autres païennes, comme la fête de l'âne ou le charivari. Mais, d'autre part, le christianisme au Moyen Âge ne se résume pas à un courant agélaste et anti-mystique. Il y a dans le Bas Moyen Âge notamment, période charnière avant la Renaissance dont Guillaume de Baskerville dans Le Nom de la rose est le représentant, l'émergence d'une théologie critique à l'égard de la dogmatique chrétienne. La théologie apophatique de saint Thomas permet également de reconsidérer le rire dans sa dimension spirituelle. Ainsi, loin d'être un antagoniste du mysticisme, le rire serait au contraire, en tant que rire de bonne foi, rire spirituel et rire de la joie, un mode d'accès singulier au divin, une sorte de disposition propre à l'expérience mystique dont la figure de saint François d'Assise témoigne particulièrement, mais avant tout celle d'Isaac, dans l'ancien testament. 


\section{De la théologie à l'expérience mystique du rire : le rire de bonne foi}

14

Parmi les théologiens du treizième siècle, saint Thomas dont la théologie négative tente de montrer que la raison ne peut qu'être servante de la foi, se rapproche le plus d'une mystique du rire. Toutefois, il s'inscrit plus dans une théologie mystique dont l'approche reste encore rationnelle que dans une expérience mystique du rire. Il est, en effet, un de ceux qui le réhabilitent rationnellement en considérant l'importance du problème du rire, notamment dans le commentaire de l'Éthique à Nicomaque par rapport auquel il réintègre l'eutrapélie aristotélicienne comme vertu du rire modéré. Cette dernière a été condamnée par saint Paul qui y a vu une disposition au rire bête et aux facéties en tout genre. En outre, saint Thomas pense que les agélastes sont des pêcheurs contre la raison. En effet, en montrant toujours un visage triste, ils sont difficiles à supporter, et désagréables aux autres : "Dans les choses humaines, tout ce qui va contre la raison est vicieux. Or, c'est aller contre la raison que d'être à charge aux autres, par exemple en ne se montrant pas joyeux et même en empêchant les autres de l'être ${ }^{22} \mathrm{En}$ d'autres termes, on peut dire que le rire est une vertu sociale et morale, il est une vertu de politesse lorsqu'il est ce savoir-vivre défini par Aristote, c'est la bonne humeur de l'homme de bonne compagnie. À travers le rire mesuré et non le rire de la moquerie que saint Thomas condamne dans la somme théologique comme étant un péché grave qui consiste à humilier ou à se réjouir de la souffrance d'autrui, est aussi réhabilitée la place de l'homme comme être naturel et raisonnable dont il faut accepter la nature faillible. À la suite d'Aristote, saint Thomas insiste finalement sur l'idée que l'origine du mal est dans l'hybris et non radicalement en l'homme sensible dont il faudrait tout condamner et rejeter comme le font les agélastes, contempteurs et hommes croyants menés par un idéalisme ascétique mortifère plus que par la raison. Au-delà du dualisme esprit/corps, saint Thomas pense en effet leur union et l'âme intellective, telle qu'il la nomme (anima intellectiva), permet de manifester que l'âme est la forme du corps. L'âme est donc le principe spirituel unitaire qui rend compte des activités de l'homme en son entier, c'est-à-dire de l'homme en tant que sujet. En ce sens pour saint Thomas, il y a une sexualité licite comme il y a un rire licite parce que l'homme est d'abord un être sensible qui du reste, est doté d'une raison pouvant limiter tout excès. Les mystiques n'ignorent pas cette double dimension lorsqu'ils font l'épreuve de l'absolu dans l'immanence, de la lumière dans l'obscurité dont le rire de la joie est la manifestation.

On pourrait d'ailleurs considérer que saint François d'Assise, frère des pauvres, est le premier mystique à saisir l'importance du rire comme signe de la foi et comme disposition singulière pour s'unir à Dieu. En complète rupture avec les pratiques monastiques du Haut Moyen Âge, il confirme ainsi avant l'heure la réflexion de Michel de Certeau concernant l'émergence de la mystique aux seizième et dix-septième siècles : «Le substantif mystique semble faire son apparition dans les milieux ou à propos des groupes qui s'éloignent le plus de l'institution théologique (...) $»^{23}$. François d'Assise fait donc du rire un idéal de spiritualité et une manière d'être dans la sainteté et la béatitude. Celui-ci « recommandait à ses disciples d'avoir toujours un visage riant (vultus hilaris) et sa première règle précisait : qu'ils prennent garde de se montrer extérieurement tristes et de sombres hypocrites, mais qu'ils se montrent joyeux dans le Seigneur, gais et agréables comme il convient $»^{24}$. La gaieté proposée par saint François d'Assise qui n'est certes pas l'hilarité excessive, est l'idée que le rire est la manifestation extérieure de la joie parfaite de l'âme qui se traduit en termes de lumière 
resplendissant sur le visage animé par le rire. Un certain rire est donc amour, lumière et charisme rayonnant, tel celui de François d'Assise qui témoigne d'une théologie positive du rire et qui confirme un modèle chrétien de joie et d'amour permettant d'être en Dieu au lieu de nous en éloigner. Si, du reste, le rire est amour et lumière, on peut très bien concevoir que la transfiguration du Christ ait été à maintes reprises l'illumination même de ce rire spirituel sur son visage. Cette conception d'un rire spirituel s'oppose à l'austérité entretenue jusqu'ici par l'Église pensant que le rire est diabolique. On assiste d'ailleurs à un changement notoire qui vient des hommes d'Église eux-mêmes. S'inscrivant dans une théologie positive, tel Jacques de Vitry, nommé évêque d'Acre en 1217, ils font " de la gaieté, une vertu chrétienne, et avec excès pour certains franciscains qui prennent à la lettre l'expression de leur fondateur : Je suis le clown du Seigneur (joculatores domini) $\gg^{25}$ Quant au franciscain Guillaume de Baskerville dont le rationalisme ne fait pas un mystique mais un théologien, son rôle principal est finalement de lutter contre tout obscurantisme et superstition entretenus par une certaine Église en défendant ceux qui en sont la cible, notamment les hérétiques. Partisan du rire, il y voit sans doute davantage un rire savant qui libère de l'ignorance et de la peur, à la manière d'un Érasme ou d'un Rabelais, qu'un saint rire, bien qu'étant disciple de la spiritualité franciscaine.

Ce rire de la joie, de l'amour et de la foi dont témoigne saint François d'Assise et pour lequel il nous faut examiner la dimension mystique, trouve sa source première dans l'ancien testament à travers la figure d'Isaac. En hébreu, Isaac se dit Sâkhaq et signifie " celui qui rira » ou « Dieu qui rit ». Isaac est donc l'incarnation symbolique du rire de la grâce divine donnée aux hommes, figure de l'espérance et premier rire de la foi comme signe de la présence divine dans l'immanence naturelle. "Rira bien qui rira le premier " pourrait-on dire à propos du rire d'Isaac. Il n'est pas le dernier et mauvais rire, rire vindicatif, mais le rire du commencement. Impulsant notre histoire, il débute avec le judaïsme comme première religion révélée concevant la transcendance et l'altérité de ce grand Autre : Dieu. Il fait événement en ceci qu'il introduit une rupture avec le rire archaïque et chaotique issu des religions païennes. Ce rire de joie (sâkhaq en hébreu), qui se distingue du mauvais rire (Iâag, à l'origine du prénom Ismaël), ce grand rire est d'abord le rire qui permet l'ouverture de la raison à la foi et à l'espérance. Il est, certes, précédé des rires suspicieux d'Abraham et de Sara, les futurs parents d'Isaac. Leur rire est d'abord le rire rationnel du refus, le rire du doute quant à la promesse divine d'enfanter, elle qui est stérile et âgée. Pourtant, dans l'expectative, elle est malgré elle conduite à espérer. Autrement dit, elle laisse advenir la confiance comme ouverture à l'altérité, comme ouverture à l'avenir. La négativité du rire de Sara prend du reste, de l'ampleur lorsqu'elle nie ce rire devant Dieu. Pourtant le rire de duplicité de Sara qui est ce qu'on pourrait nommer le mauvais rire, le rire de la finitude, devient rire de joie à l'arrivée d'Isaac. Par Isaac, on doit comprendre le rire comme don de Dieu qui transforme le rire sarcastique de Sara en rire de joie parce que ce rire est aussi le rire de la transmission de la vie par la sexualité et l'amour, rappelant par ailleurs le rire de Déméter ${ }^{26}$. Isaac est aussi le rire de la grâce comme disponibilité de l'être, accueil en soi de l'altérité divine. On peut donc entendre que cette force humaine qui doit pourtant aussi assumer sa faiblesse et sa finitude et traverser cette épreuve de l'incarnation, est dans cette ouverture à l'imprévisible, à la contingence, à l'effraction d'un réel pur échappant à la rationalité. Le rire incarne cette imprévisibilité, cette réalité absolue, cette inconnaissance, cette ouverture dans le corps et l'âme, assurant le passage entre le corps physique et le corps spirituel, assurant l'ouverture possible au divin. Par ses 
ambiguïtés (Sara et Isaac) et sa double dimension, on peut l'identifier à un trait caractéristique du mystique : sa nature paradoxale. Jeanne Guyon que cite la psychanalyste Catherine Millot en parle avec justesse : « Ces personnes, ajoute Jeanne Guyon, sont un paradoxe pour elles- mêmes et pour les autres : en elles, les contraires s'unissent. Elles sont fragiles et inaltérables, passives et hyperactives, aimantes et indifférentes, toutes dans le divin et dans le naturel, dans le vide et dans la plénitude, libres et soumises, anéanties et d'une redoutable spontanéité (...) En cela peut-être plus chrétiennes que nulle autre, prenant au pied de la lettre l'incarnation du divin, comme le fit remarquer Henri Delacroix (...) $\aleph^{27}$ Cette dualité, précise Catherine Millot : « C'est la coexistence de deux mondes étrangers l'un à l'autre, l'union " paradisiaquement assumée", non tant de deux opposés, que de deux incommensurables, que réalise l'oxymore. ${ }^{28}$ Quelle est donc cette double nature mystérieuse et secrète du rire mystique qu'il nous faut tenter de dévoiler?

Dans La divine comédie, (le Paradis, chant XXXIII), sans toutefois le nommer, Dante envisage déjà une conception mystique du rire comme ouverture au divin. Dès lors associé à l'amour que Dante porte à Béatrice, Le « saint rire » dont il parle incarne le désir érotique dont la puissance amoureuse sublime la simple dimension sexuelle et hédoniste pour l'orienter vers une force transcendante qui suppose l'abandon de soi et la perte de tout égotisme. Ainsi, telle la flèche de Cupidon, transperce-t-il le corps et l'âme du mystique, dès lors ravi par un rire qui ne lui appartient plus en propre mais auquel il s'abandonne, comme à l'être aimé. Ce rire est divin. Il est au-delà de toute merveille et nul mortel ne peut en supporter l'éclat. Dans ces conditions, on peut clairement dire que la nature ascensionnelle et non sensationnelle du rire (cette dernière faisant partie du monde sensible et de ses apparats) est la manifestation de l'abandon et de la passivité, qualifiant en propre le mystique dont l'anéantissement du moi, conduit à abolir toute dualité et toute frontière entre l'intériorité et l'extériorité. Il est ce torrent par lequel jaillit et déborde cet amour, cette joie parfaite, ce ravissement, au-delà de l'extase. Mourir à soi par le rire est par conséquent éloigné de la mortification que s'imposent tout autant religieux et mystiques, si l'on s'en tient à la théologie canonique fondée sur la pratique de l'ascèse. Quelle est donc cette ascèse singulière à la mystique qui ne renie pas le corps mais qui s'en sert comme d'un lieu de retrait et d'ouverture à Dieu ? Le corps mystique est un corps de passage dont la porosité et l'ouverture se manifestent dans le rire. Comme "pas-de-sens » pour reprendre la formule lacanienne, le rire est également rencontre, entre absurdité et sens, avec le réel pur où règne le rien.

\section{La mystique : une a-théologie rieuse}

18 C'est paradoxalement par la référence à Georges Bataille dans L'expérience intérieure, que le rire est le mieux analysé dans sa dimension mystique. Son "a-théologie rieuse ${ }^{29}$ selon Les termes de Mikkel Borch- Jacobsen semble finalement épouser - au-delà de l'aspect transgressif du rire que l'on retient habituellement dans la pensée irréligieuse et profane de Bataille, entre mysticisme et démystification - cette expérience singulière du corps et du rire chez le mystique. Dès lors, tout se passe comme si la mystique comme union à Dieu ou à une réalité absolue, était arrivée à une autonomie définitive eu égard à la théologie et à la religion institutionnelle et se découvrait en sa nature paradoxale, "athéologique et areligieuse ", mais aussi peut-être athée, réalisant ainsi ce que Catherine Millot relève des mystiques comme Jeanne Guyon : " ce sont des 
oxymores vivants $\aleph^{30}$. Georges Bataille est-il donc un oxymore vivant, un mystique athée, du moins un mystique sauvage ? N'est-il pas au contraire dans le déni de la foi et du religieux ? L'expérience intérieure, quand bien même son auteur répugnerait à parler de mystique, illustre du moins pour le rire, une expérience mystique de premier ordre. Que signifie précisément l'expérience intérieure du rire pour lui ? Elle est tout d'abord expérience souveraine qu'il qualifie d'ailleurs d'expérience mystique et dont il dégage un sens anticlérical dépourvu de toute interprétation religieuse institutionnalisée. Il précise ce que toute expérience réellement mystique devrait être : "J'entends par expérience intérieure ce que d'habitude on nomme expérience mystique : les états d'extase, de ravissement, au moins d'émotion méditée. Mais je songe moins à une expérience confessionnelle, à laquelle on a dû tenir jusqu'ici, qu'à une expérience nue, libre d'attaches, même d'origine, à quelque confession que ce soit. C'est pourquoi je n'aime pas le mot mystique. $»^{31}$

Si Bataille n'aime pas le terme mystique c'est que celui-ci fait partie du registre sémantique onto-théologique et qu'il est au préalable idéalement connoté. La théologie chrétienne y voit un esprit sans corps. Il est également rationnellement limité et défini : " Je n'aime pas non plus les définitions étroites.»" Or, il en va du terme mystique comme de celui d'expérience que Bataille voudrait souveraine, c'est-à-dire pure et détachée de tout discours rationnel, de tout concept a priori la définissant, de toute théorisation anticipée formant alors ce qu'il nomme « les présuppositions dogmatiques $\|^{33}$. C'est pourquoi à cette expérience pure, le rire répond tout particulièrement. Narguant les limites de la raison par son insolence, le rire est doute jusqu'à l'épochè. Il évide tout concept et réduit à rien toute tentative de savoir rationnel si bien qu'il oblige incessamment à revenir au degré zéro de l'expérience existentielle, à la naissance, c'est-à-dire à la venue dans le réel du sujet. La nudité et le désarroi, très proches de ce que décrivent les mystiques en termes d'abandon, d'anéantissement de soi, qualifient " cette expérience née du non-savoir ${ }^{34}$, auquel le rire renvoie le sujet en le dépouillant des oripeaux illusoires du savoir rationnel. Le principe de l'expérience est donc le nonsavoir en ce sens où l'expérience tirerait d'elle-même uniquement tout ce qu'il y a à savoir, c'est-à-dire le fait d'être : "l'expérience ne révèle rien et ne peut fonder la croyance ni en partir. L'expérience est la mise en question (à l'épreuve), dans la fièvre et l'angoisse, de ce qu'un homme sait du fait d'être. ${ }^{35}$ Elle est par conséquent négation du principe négateur de la raison qui ne peut constituer son savoir qu'en la niant ellemême. Elle est donc chez Bataille négation de la négation et s'exprime particulièrement à travers le rire comme négation accomplie de la négation dans le dépassement de la dichotomie sujet/objet : «L'expérience atteint pour finir la fusion de l'objet et du sujet, étant comme sujet non-savoir, comme objet l'inconnu. ${ }^{36}$.

Bataille ne propose donc ni une théorie de l'expérience, ni une théorie du rire. Il propose de les vivre l'un et l'autre, c'est-à-dire de les éprouver jusqu'au bout, jusqu'à l'impossible dans cette consubstantialité même de l'angoisse et du rire: "J'appelle expérience un voyage au bout du possible de l'homme. ${ }^{37}$ L'expérience et le rire, l'expérience du rire est donc l'expérience de l'extrême, l'expérience mystique par excellence. C'est pourquoi Bataille ne propose nullement une théorie du rire puisque la théorie n'en donnerait qu'une vue superficielle et ferait rater sa puissance ontologique même. C'est ce qu'il reproche précisément à Bergson en le raillant férocement : « $\mathrm{Ce}$ petit homme prudent, philosophe $!{ }^{38}$ Puis, Borch-Jacobsen citant à cet égard le début du Rire de Bergson, rajoute : 
«Ce petit homme qui parlait du petit problème, du petit mystère du rire, comment eut-il pu y voir l'énigme dernière de l'être? Prudent comme tous les philosophes depuis Aristote, il continuait à en faire l'objet de sa réflexion, au lieu de se laisser lui-même gagner par le rire, là où plus rien ne compte-ni l'objet, ni le sujet. Trop sage, le philosophe ne rit pas, même et surtout lorsqu'il théorise le rire, et il ne peut donc pas non plus en éprouver toute la gravité, tout le tragique. ${ }^{39}$

C'est que ce dernier a enfermé le rire dans une définition du comique, lui qui pourtant est le penseur de la méthode intuitive et qui se garde de ce ratage rationnel comme saisie du mouvement même de la vie dont le rire est la manifestation essentielle. Non seulement donc, le rire n'est pas objet- ce à quoi Bergson semble finalement limiter le rire- mais il n'est pas même sujet. Bataille, dans la continuité du rire de Zarathoustra, est conduit à le penser au-delà du sujet. Le rire est donc l'impropre de l'homme. Il l'excède. Le rire n'est plus le sujet mais l'être même en tant que cet être est aussi le néant.

Pour lui en effet, cette expérience mystique du rire est le rire à mort comme expérience de l'impossible. Cette expérience limite est également celle du mystique qui, au bout de la nuit obscure selon saint Jean de la Croix, rencontre le rien. Elle est d'abord la constatation de l'inanité de l'être, de son insuffisance. La chute ou déchéance de l'humanité dont le rire provient, revêt donc pour Bataille un sens ontologique. Le rire de l'être est la constatation qu'il n'est que cela, rien d'autre. Il rit parce qu'il fait luimême partie de ce naufrage ontologique dans lequel il peut s'abandonner, heureux de n'avoir plus à se soucier, le temps du rire, de sa suffisance : «Et je perds mon sérieux moi-même, en riant. Comme si c'était un soulagement d'échapper au souci de ma suffisance. $^{40}$ » Rire de l'autre, c'est par conséquent rire de cet autre moi-même, de ce semblable dans lequel je me retrouve moi-même en chutant avec lui. Le rire est alors mystique en ceci qu'il est rire partagé, il est le rire de la communion, rire eucharistique pourrait-on dire. Il implique la reconnaissance du sacrifice de l'être à l'inanité, sacrifice de l'autre en soi mais aussi de soi en l'autre. Le rire est à la fois dans et au-delà des sujets. Ni sujet, ni objet, il gagne par son émotion contagieuse et sa puissance communicative. Ainsi, ce rire qui passe partout et qui se communique de l'autre à l'autre, déchoit ontologiquement l'éventuelle supériorité du rieur se croyant nécessairement suffisant et parfait, preuve étant ici faite que le rire ne se réduit pas au rire de l'orgueil que le vieux moine Jorge juge diabolique. En ce sens, selon BorchJacobsen, le rire de Bataille est aussi un rire ontologique parce qu'il opère un renversement dans lequel la chute, symbole de l'imperfection et de la finitude devient perfection : «Le rire est le tragique inversé-surmonté, la finitude prise par le bon boutqui est évidemment le bout de l'infini, ou de Dieu. $»^{41}$ Le rire de l'être est donc un rire divin, infini, parfait parce que « Dieu n'est pas la limite de l'homme, mais la limite de l'homme est divine. $»^{42}$ Dans la perspective de Bataille, l'être n'est plus à penser en haut mais en bas et c'est par le rire que se révèle cet être ontologiquement déficient mais qui par l'épreuve de sa déficience, jusqu'à la mort, prouve qu'il est.

Mikkel Borch-Jacobsen se demande d'ailleurs légitimement à propos du rire chez Bataille : « Mais qui donc est-ce qui rit, alors ? L'être ou le néant ? L'être ne doit-il pas être, au moins, pour rire de sa propre destruction dont pourtant il se réjouit? Ne doit-il pas, pour éprouver la joie de l'anéantissement rester en vie, être vivant, être tout court ?» $»^{43}$. Qui, en effet, est-ce qui rit ? L'être même du rire au sens ontologique originel de l'être total, de l'être en tant qu'être ou bien l'homme en tant qu'être singulier ? BorchJacobsen précise à propos de ce terme "être » et du titre même qu'il a donné à son 
étude : « Le rire de l'être, cette formule un peu grandiloquente ne se trouve à vrai dire nulle part, telle quelle, dans le texte de Bataille. Elle y est pourtant impliquée, me semble-t-il de plusieurs façons. $»^{44}$ On pourrait a priori effectivement penser que la réflexion de Bataille s'inscrit davantage dans une pensée de l'immanence éradiquant ainsi tout concept métaphysique ou se jouant tout du moins de certains termes ontothéologiques dont les formules transgressives - « Dieu est un porc »-intervertissent l'ordre divin et l'ordre naturel. De son point de vue en effet, il y a inversion et séparation radicale si bien que le sacré est dans l'immanence du monde, par ce « sacré rire » notamment qui lui donne cette puissance d'élévation tandis que le profane, voire le blasphème figure dans la transcendance et le divin auxquels ce sacré rire semble mettre fin. Pour autant, on peut admettre une ambiguïté constante dans la pensée de Bataille et interroger dans le caractère profane de ses propos, un certain déni de Dieu et du religieux. Entre rire onto-théologique et rire viscéral, il semble que les extrêmes se rejoignent, pour se confondre dans cette considération d'un rire de l'être, d'un rire mystique chez Bataille. Ce sont des « oxymores vivants" pour reprendre la formule explicite de Catherine Millot.

La signification du rire mystique se trouve donc dans cette expérience de la limite là où la raison abdique. Or, cette expérience de la limite, est non seulement la confrontation de la pensée à l'impossible et impensable mort d'où résulte le rire mais est surtout chez Bataille l'expérience paradoxale la plus tragique et profonde de l'identité de l'être et de la mort. Celle-ci se révèle précisément dans ce sentiment fulgurant de l'inanité de l'être. Borch-Jacobsen cite à ce propos la préface à Madame Edwarda : «Ce dont ce grand rire nous détourne, que suscite la plaisanterie licencieuse, est l'identité du plaisir extrême et de l'extrême douleur : l'identité de l'être et de la mort, du savoir s'achevant sur cette perspective éclatante et de l'obscurité définitive. De cette vérité, sans doute, nous pourrons finalement rire mais cette fois d'un rire absolu, qui ne s'arrête pas au mépris de ce qui peut être répugnant, mais dont le dégoût nous enfonce. ${ }^{45} \mathrm{Ce}$ rire à mort, ce rire qui rit à partir du paradoxe de l'être comme néant et qui arrive à en rire, est le rire de l'expérience de l'impossible, c'est-à-dire littéralement de ce qui s'éprouve jusqu'à la limite de l'insupportable. Ce que dit Bataille de cette expérience limite dont le corps est le lieu et qui consiste à abolir les résistances du moi, présente de troublantes similitudes avec ce que relate Catherine Millot de la mystique Jeanne Guyon et de l'ascèse la préparant à l'anéantissement de son propre moi.

Elle «se guide sur ses plus fortes répulsions pour les attaquer et les vaincre. Les crachats lui font horreur. Leur simple vue lui donne envie de vomir. "Il me fallut un jour que j'étais seule et que j'en aperçus un, le plus vilain que j'aie jamais vu, mettre ma bouche et ma langue dessus : l'effort que je me fis fut si étrange que je ne pouvais en revenir et j'eus des soulèvements de cour si violents que je crus qu'il se romprait en moi quelques veines, et que je vomirais le sang. Je fis cela tout autant de temps que mon cour y répugna, ce qui fut assez long (...) "et elle précise qu'elle n'obéissait pas à un plan délibéré mais que Dieu l'y poussait et ne lui passait rien. $»^{46}$

Dans l'expérience mystique, en effet, Dieu est souverain, tout comme le rire de l'être pour Bataille. Il est la marque de la supériorité, non au sens d'une volonté de pouvoir sur autrui rejoignant alors la théorie de la supériorité du rieur riant de l'autre mais au sens premier du terme issu de l'étymologie latine supraneus, signifiant supérieur, haut, noble, élevé. La souveraineté du rire de l'être est cette puissance d'être au-delà du sujet, capable de rire de lui-même et de son propre rire, puissance qui excèderait l'être luimême dans cette épreuve du passage de l'être au néant. La souveraineté du rire est donc la manifestation du réel absolu, au-delà de l'homme. Elle justifie cette pensée de 
Bataille extraite du Le coupable que cite Borch-Jacobsen selon laquelle finalement le rire n'est plus le propre de l'homme mais bien plutôt l'homme le propre du rire auquel il doit advenir : «Toujours j'ai reculé devant l'échéance : j'avais peur d'être ce que j'étais : le rire même ! $(\mathrm{V}, 334)$ Ou encore : Je ne suis à la vérité que le rire qui me prend. $»^{47}(\mathrm{~V}$, 364) De la même manière, dans l'expérience mystique, non seulement le sujet est pris et ne s'appartient plus mais il n'est plus qu'à Dieu et en Dieu. "L'âme n'est plus au-dedans mais elle est toute en Dieu $»^{48}$ relate Jeanne Guyon. L'expérience mystique est réalisée, « la vie parfaite » est là : union avec Dieu, fusion joyeuse en lui, béatitude. Le rire comme puissance de vie, comme puissance de l'être, comme puissance divine, l'exprime en ce qu'il excède et dépasse l'homme en tant que sujet corporel, moral psychique, intellectuel. S'il est une surpuissance ou puissance excessive que le sujet seul ne pourrait contenir, c'est parce qu'il vient de cette rencontre entre le réel pur et la psyché, point de rencontre ne générant pas un mode hallucinatoire et un mode représentatif illusoire, mais une distanciation lucide. Il se situe simultanément dans et hors du sujet comme puissance extatique. Il s'élabore dans une sorte de mouvement qui s'origine dans le corps et s'expulse hors de lui comme une catharsis des humeurs. Il opère donc un détachement par rapport à l'immédiateté de l'expérience vécue. Il est, ce que Lacan nomme "l'extime " à propos de l'expérience limite du corps chez le mystique où l'intime est passé à l'extérieur tandis que l'extérieur est tout entré dans le dedans. Le rire ouvre le corps et l'âme, il les dilate par son souffle et insuffle une joie indicible que jeanne Guyon, en parlant de gaieté, recommandera à Fénélon dont l'absence de désir et la sécheresse empêchent en lui « l'entière largeur et étendue que Dieu veut qui y soit $\star^{49}$. Elle l'exhorte donc à se « laisser dilater en toutes manières $»^{50} \mathrm{et}$ à «demeurer libre et gai $»^{51}$ car, conclut Catherine Millot : «tandis que la tristesse resserre, la joie dilate, elle est de Dieu. ${ }^{52}$

Il apparait dès lors, que loin d'être anti-mystique et diabolique selon une certaine théologie chrétienne, le rire présente au contraire tous les attributs de l'âme et du corps qui disposent le mystique à cet abandon de soi. Faisant voler en éclats ce qui reste d'orgueil, d'attachement à soi, il est, par l'humour notamment, ce retour à l'humus, à la simplicité enfantine de l'âme, à l'humilité nécessaire pour constater l'inanité de l'être. (Il y aurait peut-être en effet une origine étymologique commune entre l'humeur dont dérive le terme humour et l'humus dont dérive le terme humilité). Le rire est donc l'expérience intérieure la plus originelle, celle qui définit finalement le mystique dont la rencontre avec l'absolu auquel il s'abandonne, le dépouille de tout et le met face à l'angoisse $\mathrm{du}$ rien. C'est étrangement hors du champ institutionnel religieux, précisément dans l'athéologie rieuse de Bataille, que l'on est amené à comprendre toutes les dimensions du rire jusqu'au rire mystique. Il est, lui aussi, à sa manière, un oxymore, tout comme le mystique selon les termes de Catherine Millot. On conclura sur cette remarque qu'on pourrait nous objecter concernant l'absence de référence au sourire qui, dans les figures saintes du christianisme, y a davantage sa place. C'est que, traitant du rire comme disposition mystique singulière, on retiendra que leur nature commune se manifeste dans une sorte d'excès, c'est-à-dire non dans la transgression mais dans le dépassement de soi. Que celle-ci soit extatique ou extraordinaire par l'ascèse qu'elle implique, la particularité de l'expérience mystique, est cette sortie de soi auquel le sourire, le « sous-rire» (subrisus en latin), expression de la retenue et de la sagesse, expression de la raisonnable sainteté, expression de la convenance, ne peut pas tout à fait conduire. Il lui faut raison garder tandis que, dans l'expérience mystique, du rire notamment, il faut raison lâcher. 


\section{BIBLIOGRAPHIE}

Georges BATAILLE, L'expérience intérieure, Paris, Gallimard, 1943, 1954.

Michel DE CERTEAU, La fable mystique, Paris, Gallimard, 1982.

Umberto ECO, Le nom de la rose, trad. franç., J.N.Schifano, Paris, Grasset, 1982.

Michel HULIN, La mystique sauvage, Paris, PUF, 2008.

Jacques LE GOFF, Un autre Moyen Âge (Le rire dans les règles monastiques du haut Moyen Âge), Paris, coll. « Poche », Gallimard, 1999.

Catherine MILLOT, La vie parfaite, Paris, Gallimard, 2006.

Georges MINOIS, Histoire du rire et de la dérision, Paris, Fayard, 2000. Pseudo-Denys L'ARÉOPAGITE, Théologie mystique, Trad. M. Cassingena, Paris, Éditions J.P Migne.

Articles

Mikkel BORCH-JACOBSEN, « Bataille et le rire de l'être », Revue critique, Quatre essais sur le rire, Janvier-février 1988.

Jacques LE GOFF, « Rire au Moyen Âge », Les cahiers du centre de recherches historiques, Mars 1989.

\section{NOTES}

1. G. Bataille, Le coupable, Euvres complètes V, Paris, Gallimard, 1973, V 364.

2. U. Eco, Le Nom de la Rose, trad. Franç. J.N.Schifano, Paris, Grasset, 1982, p. 592.

3. M. Hulin, La mystique Sauvage, Paris, PUF, 2008.

4. Pseudo-Denys l'Aréopagite, Théologie mystique, trad. Franç. M. Cassingena, Paris, Éditions J.P Migne, p.35.

5. Ibid., p. 22.

6. Le Nom de la rose, est aussi un film franco-germano-italien réalisé par Jean-Jacques Annaud et sorti en 1986, d'après le roman éponyme d'Umberto Eco. C'est dans une des joutes oratoires entre Guillaume de Baskerville et le vieux moine Jorge que cette parole est prononcée.

7. Jacques Le Goff, « Rire au Moyen Âge », article présenté en " séminaire du centre » et extrait des cahiers du centre de recherches historiques, Mars 1989. Cet article renvoie à l'ouvrage de Jacques le Goff, Un autre Moyen Âge (Le rire dans les règles monastiques du haut Moyen Âge), Paris, coll. « Poche » Gallimard, 1999.

8. Ibid.

9. G.Minois, Histoire du rire et de la dérision, Paris, Fayard, 2000, p. 127.

10. Ibid., p.127.

11. J. Le Goff, op. cit.

12. Ibid.

13. G. Minois, op. cit., p. 21.

14. Ibid., p. 211.

15. Ibid., p.212.

16. Ibid., p.212.

17. Ibid., p.212.

18. Ibid., p.213.

19. M. de Certeau, La fable mystique, Paris, Gallimard, 1982, pp.58-65. 
20. Selon Michel Hulin, les expériences mystiques peuvent se produire hors de la sphère religieuse. D'où l'expression « mystique sauvage ».

21. M. de Certeau, Ibid., p. 148-149.

22. G. Minois, Ibid., p. 209.

23. M.de. Certeau, Ibid., p.148-149.

24. G. Minois, Ibid., p.191.

25. G. Minois, Ibid., p. 191.

26. Le mythe de Baubô raconte en effet, comment Déméter qui vient de perdre sa fille Perséphone et qui souffre de mélancolie, revient à la vie grâce à Baubô. Cette dernière, en faisant le geste obscène de retrousser son vêtement, laisse voir un sexe riant dont la version orphique raconte : « il y avait l'enfant Iacchos qui riait sous les jupes de Baubô. Il l'agita de la main. Alors elle sourit, la déesse, elle sourit en son cœur (...) ». Une des interprétations possible de la présence de cet enfant sous les jupes de Baubô est de transmettre à Déméter que la vie continue pour elle parce qu'elle peut encore la donner. Son rire est donc dans une certaine mesure, le rire de la guérison par la foi recouvrée.

27. C. Millot, La vie parfaite, Paris, Gallimard, p. 69.

28. Ibid., p.69.

29. M. Borch-Jacobsen, «Bataille et le rire de l'être », Revue critique, Quatre essais sur le rire, Janvier-février 1988, p. 31.

30. C. Millot, Ibid., p. 69.

31. G. Bataille, L'expérience intérieure, Paris, Gallimard, 1943 et 1954, p. 15.

32. Ibid.

33. Ibid.

34. Ibid.

35. Ibid., p. 16.

36. Ibid., p. 21.

37. Ibid., p. 19.

38. Ibid., p. 80.

39. M. Borch-jacobsen, « Bataille et le rire de l'être », op. cit., p. 21.

40. Ibid.

41. Ibid., p. 29.

42. Ibid., p. 31.

43. Mikkel Borch-Jacobsen, «Bataille et le rire de l'être », op. cit., p. 24 .

44. Ibid., p. 23.

45. Ibid., p. 20

46. C. Millot, Ibid., p. 34.

47. M. Borch-Jacobsen, Ibid., p. 24.

48. C. Millot, Ibid., p. 59.

49. Ibid., p.80.

50. Ibid., p.80.

51. Ibid., p.80.

52. Ibid., p.80. 


\section{RÉSUMÉS}

Les théologiens et mystiques chrétiens, notamment au Moyen Âge, ne sont guère enclins à approuver le rire. Dans ses manifestations corporelles hybristiques, ils voient le diable incarné. Dès lors, est-il le rire anti-mystique, le rire de mauvaise foi ? Pourtant, si l'on s'en tient à la figure biblique d'Isaac ou à saint François d'Assise, le rire est un don de Dieu. Il est joie et foi. Disposition singulière du corps que le mystique éprouve, il conduit à l'extase et à l'abandon de soi. C'est paradoxalement à travers " l'athéologie rieuse " de Georges Bataille que le rire est le mieux analysé dans sa dimension mystique. Finalement, comprendre le rire et la mystique, ne suppose-t-il pas de se détacher d'une représentation ascétique désincarnée qui conditionne pour partie la théologie chrétienne?

Christian theologians and mystics, especially in the Middle Ages, are hardly inclined to approve laughter. In its hubristic bodily manifestations, they see the devil incarnate. So, is laughter in bad faith anti-mystical laughter? Yet, if we stick to the biblical figure of Isaac or St. Francis of Assisi, laughter is a gift from God. It is joy and faith. A singular disposition of the body that mystic experiences lead to ecstasy and self-denial. Paradoxically, it is through Georges Bataille's "Laughing Atheology" that laughter is best analysed in its mystical dimension. And last but not least, does it not imply that laughter and mysticism imply detachment from a disembodied ascetical representation that partly conditions Christian theology?

\section{AUTEUR}

\section{SYLVIE PAILLAT}

Sylvie Paillat, docteur en philosophie, membre du CRICES, chargée de cours à l'ICES et professeur en lycée, est l'auteur d'une thèse : Métaphysique du rire, (L'Harmattan, mai 2014). Le problème du rire y est abordé dans ce qui le lie intrinsèquement à la rationalité philosophique. Il est un moyen de repenser la métaphysique occidentale en s'interrogeant sur les fondements de sa nature psycho-rationnelle. Ses champs d'application sont multiples : théologique, épistémologique, esthétique, éthique, politique. Quelques articles sur le même sujet ont également été publiés dans Le Philosophoire : « Esthétique du rire » et «L'intelligence du rire ou la bêtise feinte ». 